\title{
The Case Of Alpha Inc.: A study In Forecasting And Valuation
}

Merouane Lakehal-Ayat, PhD, St. John Fisher College, USA

\begin{abstract}
ALPHA Inc., a manufacturing entity, hired James to help put their records in for 2006 and 2007. James completed the financial statements to gain an understanding of the accounting transactions and financial results. In addition, ALPHA Inc. requested a five-year forecast for future financing and wanted a valuation of the company for possible sale or merger. After students complete the accounting relationships, financial analysis, forecast and valuation, they will have a solid background to complete the financial section of a business plan. This case is most appropriate for an upper division accounting or finance course.
\end{abstract}

Keywords: Financial Statement Analysis- Forecasting- Valuation

\section{EXECUTIVE SUMMARY}

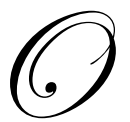

n Tuesday January 4, 2009, ALPHA Inc., a manufacturing entity, hired James Savage, a young, astute and ambitious consultant. At the meeting, James received a simple chart of accounts, an Opening Day Balance Sheet, First Year Balance sheet, and First year Income Statement.

After James compiled an income statement, balance sheet, cash flow statement and financial ratios for 2005 through 2006, he needed to develop a five-year forecast for 2008 to 2012 and a valuation under three different methods. In creating the forecast, ALPHA decided to purchase a lathe and drill press to improve operations.

\section{THE CASE}

On Tuesday morning, ALPHA hired James Savage, a young, astute and ambitious consultant. At the first meeting with the president, George Key, James learned that the company had no solid financial records and lacked operational understanding for the past three years. George revealed that financial records were limited and not maintained for the last three years.

In order to gain an understanding of ALPHA's operations, James compiled: a 2005 through 2007 Balance Sheet (see Table 1), a 2005 through 2007 common size balance sheet (see Table 2), a 2005 through 2007 statement of retained earnings (see Table 3), a 2005 through 2007 Income Statement (see Table 4), 2006 and 2007 cash flow statement (see Table 5), and 2005 through 2007 ratio analysis table (see Table 6). However, James understood that he still had the hardest part of the consulting job to complete - the forecast and valuation.

After the financial ratios, James needed to complete a five-year forecast to help in determining the valuation of ALPHA, a "C" Corporation. George noted the forecast should be on an annual basis for 2008 through 2012. George wanted to make sure that James made the correct assumptions in the forecast and decided that he would like to see a list of all assumptions. George stated, "Your assumptions must be logical, reasonable, and defensible for each component forecasted in each statement. You cannot just make changes in numbers without justification."

George mentioned to James that he was planning to finance two new pieces of equipment in 2008 and the following information was available for the forecast: 
The first piece of equipment would be a new lathe with XYZ cutting and shaping. The life of the machine would be 510 years and cost $\$ 55,000$. The second purchase, a Drill Press, would cost $\$ 35,000$ and have a useful life of 5-10 years.

George wanted to see a repayment schedule with total payments, interest and principal for each piece of equipment. However, George did not know what an acceptable rate of interest or repayment time would be for the equipment. In addition, George wanted James to answer the following questions about the loans:

- Will these loans be secured or unsecured? If secured, what type of collateral would be needed for the loans?

- What will be the impact on the financial statements for these two pieces of equipment?

In addition, George mentioned that he would like to take out $\$ 100,000$ in dividends each year over the next five years to invest in other personal projects.

Lastly, James needed to figure out a value for ALPHA, per George's request. James thought that the following methods were appropriate: Book Value method (Assets-Liabilities), Liquidation Valuation method (i.e. $60 \%$ of accounts receivable, $25 \%$ of equipment, and $20 \%$ of inventory) and Free Cash flow Method that included the following data:

- $\quad$ Start with Earnings before Interest and Taxes

- $\quad$ Add back owner's salary, depreciation, personal expense

- $\quad$ Subtract the new salary for the owners, new equipment purchases or payments for new equipment, and investment in inventory to determine free cash flow

- A good rate of return on the investment of the company would range from 20 to $35 \%$ because the company is privately held.

Do not forget:

- To forecast for five years

- $\quad$ Discount the terminal value plus all five years back to the present value; subtract debt to be paid and capital gain taxes to determine a final value for the company.

James must complete the following items for George as part of his consulting contract:

\section{$\underline{\text { PART I }}$}

that:

Complete a five year forecast of the income statement, balance sheet and cash flow statement for ALPHA

- Incorporates the two loan payments (including the repayment term and interest rate) to show the impact of the loans on the company operations

- Uses information about the industry to shape the forecast

- $\quad$ Provides a list of assumptions for the forecast

- Includes a term sheet that helps structures the two loans on the equipment that includes the interest rate, repayment term, schedule of annual repayment, and determines collateral

\section{$\underline{\text { PART II }}$}

Determine the value for ALPHA Inc. under the following methods:

- $\quad$ Book value

- $\quad$ Liquidation Value

- $\quad$ Free Cash Flow 
Some questions that James had before rendering his decision to George were: What are the advantages and disadvantages of each method? Which method best represents the firm's value?

Remember that the key to a good valuation is a list of assumptions needed in determining the firm value.

\section{AUTHOR INFORMATION}

Dr. Merouane Lakehal-Ayat is a full Professor of Finance at St. John Fisher College, Rochester, New York. He has taught at St. John Fisher College since 1986. Dr. M. Lakehal-Ayat holds a PhD in International Economics and Finance, MBA (Finance) and Masters in International Management and Economics from the University of DenverColorado and a JD and BSBA from the University of Algiers-Algeria. Dr. M. Lakehal-Ayat was awarded Fulbright Fellowships to Malaysia, Poland and Thailand during the 2001-2009 period. He is presently a Visiting Fellow at UTM Malaysia. E-mail: lakehal@sjfc.edu 
Table 1 - Historical Balance Sheet

Beg 2005

Assets

\section{Current Assets}

Cash

Accts Rec

Inventory

Total CA

Propert, Plant and Equipment

Equipment \& Fixtures

Transportation Equipment

Accum Depr

Total PP\&E

Total Assets

Liabilities

\section{Current Liabilities}

Accts pay

Wages payable

FIT Payable

Total CL

Long-term Liabilities

Loan Payable

Total Long-term Liab

Total Liab

Equity

Common Stock

Retained Earnings

Total Equity

Total Liab \& Eq
2005

2006

2007

\begin{tabular}{rrrr}
$\$ 249,993$ & $\$ 367,425$ & $\$ 523,717$ & $\$ 774,371$ \\
$\$ 36,283$ & $\$ 42,549$ & $\$ 52,281$ & $\$ 50,027$ \\
$\$ 17,555$ & $\$ 10,952$ & $\$ 16,450$ & $\$ 17,925$ \\
\hline$\$ 303,831$ & $\$ 420,926$ & $\$ 592,448$ & $\$ 842,323$
\end{tabular}

\begin{tabular}{rrrr}
$\$ 372,789$ & $\$ 415,678$ & $\$ 464,967$ & $\$ 513,221$ \\
$\$ 30,769$ & $\$ 30,769$ & $\$ 30,769$ & $\$ 30,769$ \\
$(\$ 270,816)$ & $(\$ 326,757)$ & $(\$ 390,885)$ & $(\$ 464,939)$ \\
\hline$\$ 132,742$ & $\$ 119,690$ & $\$ 104,851$ & $\$ 79,051$ \\
& & & \\
\hline \hline 436,573 & $\$ 540,616$ & $\$ 697,299$ & $\$ 921,374$ \\
\hline
\end{tabular}

\begin{tabular}{rrrr}
$\$ 32,568$ & $\$ 71,711$ & $\$ 127,255$ & $\$ 155,914$ \\
$\$ 13,524$ & $\$ 11,764$ & $\$ 13,274$ & $\$ 15,712$ \\
$\$ 2,853$ & $\$ 1,452$ & $\$ 2,740$ & $\$ 4,236$ \\
\hline$\$ 48,945$ & $\$ 84,927$ & $\$ 143,269$ & $\$ 175,862$
\end{tabular}

\begin{tabular}{llll}
$\$ 322,188$ & $\$ 357,188$ & $\$ 360,464$ & $\$ 412,108$ \\
\hline$\$ 322,188$ & $\$ 357,188$ & $\$ 360,464$ & $\$ 412,108$ \\
\hline$\$ 371,133$ & $\$ 442,115$ & $\$ 503,733$ & $\$ 587,970$
\end{tabular}

\begin{tabular}{rrrr}
$\$ 100$ & $\$ 100$ & $\$ 100$ & $\$ 100$ \\
$\$ 65,340$ & $\$ 98,401$ & $\$ 193,466$ & $\$ 333,304$ \\
\hline$\$ 65,440$ & $\$ 98,501$ & $\$ 193,566$ & $\$ 333,404$ \\
& & & \\
\hline$\$ 436,573$ & $\$ 540,616$ & $\$ 697,299$ & $\$ 921,374$ \\
\hline \hline
\end{tabular}


Table 2 - Historical Common Size Balance Sheet

\begin{tabular}{|c|c|c|c|c|c|c|c|}
\hline Balance Sheet & Beg 2005 & 2005 & 2006 & 2007 & Beg 05-06 & $05-06$ & 06-07 \\
\hline \multicolumn{8}{|l|}{ Assets } \\
\hline \multicolumn{8}{|l|}{ Current Assets } \\
\hline Cash & $57.3 \%$ & $68.0 \%$ & $75.1 \%$ & $84.0 \%$ & $47.0 \%$ & $42.5 \%$ & $47.9 \%$ \\
\hline Accts Rec & $8.3 \%$ & $7.9 \%$ & $7.5 \%$ & $5.4 \%$ & $17.3 \%$ & $22.9 \%$ & $-4.3 \%$ \\
\hline Inventory & $4.0 \%$ & $2.0 \%$ & $2.4 \%$ & $1.9 \%$ & $-37.6 \%$ & $50.2 \%$ & $9.0 \%$ \\
\hline Total CA & $69.6 \%$ & $77.9 \%$ & $85.0 \%$ & $91.4 \%$ & $38.5 \%$ & $40.7 \%$ & $42.2 \%$ \\
\hline \multicolumn{8}{|c|}{ Propert, Plant and Equipment } \\
\hline Equipment \& Fixtures & $85.4 \%$ & $76.9 \%$ & $66.7 \%$ & $55.7 \%$ & $11.5 \%$ & $11.9 \%$ & $10.4 \%$ \\
\hline Transportation Equipment & $7.0 \%$ & $5.7 \%$ & $4.4 \%$ & $3.3 \%$ & $0.0 \%$ & $0.0 \%$ & $0.0 \%$ \\
\hline Accum Depr & $-62.0 \%$ & $-60.4 \%$ & $-56.1 \%$ & $-50.5 \%$ & $20.7 \%$ & $19.6 \%$ & $18.9 \%$ \\
\hline Total PP\&E & $30.4 \%$ & $22.1 \%$ & $15.0 \%$ & $8.6 \%$ & $-9.8 \%$ & $-12.4 \%$ & $-24.6 \%$ \\
\hline Total Assets & $100.0 \%$ & $100.0 \%$ & $100.0 \%$ & $100.0 \%$ & $23.8 \%$ & $29.0 \%$ & $32.1 \%$ \\
\hline
\end{tabular}

Liabilities

\section{Current Liabilities}

Accts pay

Wages payable

FIT Payable

Total CL

\begin{tabular}{rrrrrrr}
$7.5 \%$ & $13.3 \%$ & $18.2 \%$ & $16.9 \%$ & $120.2 \%$ & $77.5 \%$ & $22.5 \%$ \\
$3.1 \%$ & $2.2 \%$ & $1.9 \%$ & $1.7 \%$ & $-13.0 \%$ & $12.8 \%$ & $18.4 \%$ \\
$0.7 \%$ & $0.3 \%$ & $0.4 \%$ & $0.5 \%$ & $-49.1 \%$ & $88.7 \%$ & $54.6 \%$ \\
\hline $11.2 \%$ & $15.7 \%$ & $20.5 \%$ & $19.1 \%$ & $73.5 \%$ & $68.7 \%$ & $22.7 \%$
\end{tabular}

\section{Long-term Liabilities}

Loan Payable

Total Long-term Liab

Total Liab

\begin{tabular}{rrrrrrr}
$73.8 \%$ & $66.1 \%$ & $51.7 \%$ & $44.7 \%$ & $10.9 \%$ & $0.9 \%$ & $14.3 \%$ \\
\hline $73.8 \%$ & $66.1 \%$ & $51.7 \%$ & $44.7 \%$ & $10.9 \%$ & $0.9 \%$ & $14.3 \%$ \\
\hline $85.0 \%$ & $81.8 \%$ & $72.2 \%$ & $63.8 \%$ & $19.1 \%$ & $13.9 \%$ & $16.7 \%$
\end{tabular}

Equity

Common Stock

Retained Earnings

Total Equity

Total Liab \& Eq

\begin{tabular}{rrrrrrr}
$0.0 \%$ & $0.0 \%$ & $0.0 \%$ & $0.0 \%$ & $0.0 \%$ & $0.0 \%$ & $0.0 \%$ \\
$15.0 \%$ & $18.2 \%$ & $27.7 \%$ & $36.2 \%$ & $50.6 \%$ & $96.6 \%$ & $72.3 \%$ \\
\hline $15.0 \%$ & $18.2 \%$ & $27.8 \%$ & $36.2 \%$ & $50.5 \%$ & $96.5 \%$ & $72.2 \%$ \\
\hline $100.0 \%$ & $100.0 \%$ & $100.0 \%$ & $100.0 \%$ & $23.8 \%$ & $29.0 \%$ & $32.1 \%$ \\
\hline \hline
\end{tabular}


Table 3 - Historical Retained Earnings Statement

\begin{tabular}{|c|c|c|c|}
\hline & 2005 & 2006 & 2007 \\
\hline Beginning Retained Earnings & $\$ 65,340$ & $\$ 98,401$ & $\$ 193,466$ \\
\hline Plus Net Income & $\$ 33,061$ & $\$ 95,065$ & $\$ 139,838$ \\
\hline Subtotal & $\$ 98,401$ & $\$ 193,466$ & $\$ 333,304$ \\
\hline Less: Dividends & $\$ 0$ & $\$ 0$ & $\$ 0$ \\
\hline Ending Retained Earnings & $\$ 98,401$ & $\$ 193,466$ & $\$ 333,304$ \\
\hline
\end{tabular}

Table 4 - Historical Income Statement

\begin{tabular}{|c|c|c|c|c|c|c|c|c|}
\hline Income Statement & 2005 & $\%$ & 2006 & $\%$ & 2007 & $\%$ & 05-06 & 06-07 \\
\hline Gross Sales & $\$ 1,038,569$ & $100.0 \%$ & $\$ 1,239,391$ & $100.0 \%$ & $\$ 1,060,627$ & $100.0 \%$ & $19.3 \%$ & $-14.4 \%$ \\
\hline \multicolumn{9}{|l|}{ Cost of goods sold } \\
\hline Material & $\$ 106,177$ & $10.2 \%^{\prime}$ & $\$ 117,847$ & $9.5 \%^{\prime \prime}$ & $\$ 65,401$ & $6.2 \%$ & $11.0 \%$ & $-44.5 \%$ \\
\hline Shop tools & $\$ 31,355$ & $3.0 \%$ & $\$ 35,945$ & $2.9 \%^{\mathbf{r}}$ & $\$ 66,876$ & $6.3 \%$ & $14.6 \%$ & $86.1 \%$ \\
\hline Direct Labor & $\$ 312,400$ & $30.1 \%^{\prime \prime}$ & $\$ 330,888$ & $26.7 \%^{\prime \prime}$ & $\$ 328,848$ & $31.0 \%$ & $5.9 \%$ & $-0.6 \%$ \\
\hline Cost of Goods Sold & $\$ 449,932$ & $43.3 \%$ & $\$ 484,680$ & $39.1 \%$ & $\$ 461,125$ & $43.5 \%$ & $7.7 \%$ & $-4.9 \%$ \\
\hline Gross Margin & $\overrightarrow{\$ 588,637}$ & $56.7 \%$ & $\overline{\$ 754,711}$ & $60.9 \%$ & $\$ 599,502$ & $56.5 \%$ & $28.2 \%$ & $-20.6 \%$ \\
\hline \multicolumn{9}{|l|}{ Operating Expenses } \\
\hline Officer's Salary & $\$ 285,246$ & $27.5 \%^{\prime}$ & $\$ 361,019$ & $29.1 \%^{\boldsymbol{r}}$ & $\$ 164,418$ & $15.5 \%$ & $26.6 \%$ & $-54.5 \%$ \\
\hline Payroll Taxes & $\$ 31,922$ & $3.1 \%^{\prime}$ & $\$ 28,925$ & $2.3 \%^{\mathbf{r}}$ & $\$ 31,021$ & $2.9 \%$ & $-9.4 \%$ & $7.2 \%$ \\
\hline Rent & $\$ 23,268$ & $2.2 \%$ & $\$ 23,268$ & $1.9 \%^{\prime \prime}$ & $\$ 25,689$ & $2.4 \%$ & $0.0 \%$ & $10.4 \%$ \\
\hline Depreciation & $\$ 55,941$ & $5.4 \%^{\prime \prime}$ & $\$ 64,128$ & $5.2 \%^{\prime \prime}$ & $\$ 74,054$ & $7.0 \%$ & $14.6 \%$ & $15.5 \%$ \\
\hline Insurance & $\$ 29,360$ & $2.8 \%^{\prime \prime}$ & $\$ 35,539$ & $2.9 \%^{\prime \prime}$ & $\$ 28,309$ & $2.7 \%$ & $21.0 \%$ & $-20.3 \%$ \\
\hline Telephone and Utilities & $\$ 14,235$ & $1.4 \%^{\prime \prime}$ & $\$ 16,148$ & $1.3 \%^{\prime \prime}$ & $\$ 12,232$ & $1.2 \%$ & $13.4 \%$ & $-24.3 \%$ \\
\hline Travel and Entertainment & $\$ 6,789$ & $0.7 \%^{\prime \prime}$ & $\$ 7,845$ & $0.6 \%^{\prime \prime}$ & $\$ 9,377$ & $0.9 \%$ & $15.6 \%$ & $19.5 \%$ \\
\hline Repairs and Maintenance & $\$ 37,640$ & $3.6 \%^{\prime \prime}$ & $\$ 49,443$ & $4.0 \%^{\mathbf{r}}$ & $\$ 21,394$ & $2.0 \%$ & $31.4 \%$ & $-56.7 \%$ \\
\hline Office Expenses & $\$ 24,871$ & $2.4 \%^{\prime \prime}$ & $\$ 26,875$ & $2.2 \%^{\prime \prime}$ & $\$ 37,981$ & $3.6 \%$ & $8.1 \%$ & $41.3 \%$ \\
\hline Auto Expense & $\$ 7,430$ & $0.7 \%^{\prime \prime}$ & $\$ 8,336$ & $0.7 \%^{\prime \prime}$ & $\$ 9,049$ & $0.9 \%$ & $12.2 \%$ & $8.6 \%$ \\
\hline Total Operating Expense & $\overline{\$ 516,702}$ & $49.8 \%$ & $\overline{\$ 621,526}$ & $50.1 \%$ & $\$ 413,524$ & $39.0 \%$ & $20.3 \%$ & $-33.5 \%$ \\
\hline Income from Operations & $\$ 71,935$ & $6.9 \%$ & $\$ 133,185$ & $10.7 \%$ & $\$ 185,978$ & $17.5 \%$ & $85.1 \%$ & $39.6 \%$ \\
\hline \multicolumn{9}{|c|}{ Other Income and expense } \\
\hline Interest Expense & $\$ 9,532$ & $0.9 \%$ & $\$ 8,778$ & $0.7 \%$ & $\$ 14,186$ & $1.3 \%$ & $-7.9 \%$ & $61.6 \%$ \\
\hline Earnings before taxes & $\$ 62,403$ & $6.0 \%$ & $\$ 124,407$ & $10.0 \%$ & $\$ 171,792$ & $16.2 \%$ & $99.4 \%$ & $38.1 \%$ \\
\hline Taxes & $\$ 29,342$ & $2.8 \%$ & $\$ 29,342$ & $2.4 \%$ & $\$ 31,954$ & $3.0 \%$ & $0.0 \%$ & $8.9 \%$ \\
\hline Net income & $\$ 33,061$ & $3.2 \%$ & $\$ 95,065$ & $7.7 \%$ & $\$ 139,838$ & $13.2 \%$ & $187.5 \%$ & $47.1 \%$ \\
\hline
\end{tabular}


Table 5 - Historical Cash Flow Statement

Cash Flow Statement Ope rating:

Net Income

+ Depreciation \& Amortization

-Incr/+Decr in Receivables

-Incr/+Decr in Inventory

-Incr/+Decr in Other Current Assets

+ Incr/-Decr in Accounts Payable

+ Incr/-Decr in Other Liabilities

$=$ Cash From Operations

Investing:

-Capital Expenditures

-Increase in Investments

-Purchases of Intangibles

-Increase in Other Assets

$=$ Cash From Investing

\section{Financing:}

+Increase in Debt

-Dividends Paid on Preferred

+Increase in Pref. Stock

-Dividends Paid on Common

+/-Net Issuance of Common Stock

+/-Clean Surplus Plug (Ignore)

$=$ Cash From Financing

Net Change in Cash

+ Beginning Cash Balance

$=$ Ending Cash Balance
2006

2007

\begin{tabular}{rr}
$\$ 95,065$ & $\$ 139,838$ \\
$\$ 64,128$ & $\$ 74,054$ \\
$(\$ 9,732)$ & $\$ 2,254$ \\
$(\$ 5,498)$ & $(\$ 1,475)$ \\
$\$ 0$ & $\$ 0$ \\
$\$ 55,544$ & $\$ 28,659$ \\
$\$ 2,798$ & $\$ 3,934$ \\
\hline 202,305 & $\$ 247,264$
\end{tabular}

\begin{tabular}{rr}
$(\$ 49,289)$ & $(\$ 48,254)$ \\
$\$ 0$ & $\$ 0$ \\
$\$ 0$ & $\$ 0$ \\
$\$ 0$ & $\$ 0$ \\
\hline$(\$ 49,289)$ & $(\$ 48,254)$
\end{tabular}

\begin{tabular}{rr}
$\$ 3,276$ & $\$ 51,644$ \\
$\$ 0$ & $\$ 0$ \\
$\$ 0$ & $\$ 0$ \\
$\$ 0$ & $\$ 0$ \\
\hline$\$ 3,276$ & $\$ 51,644$ \\
$\$ 0$ & $\$ 0$ \\
\hline$\$ 3,276$ & $\$ 51,644$
\end{tabular}

\begin{tabular}{ll}
$\$ 156,292$ & $\$ 250,654$ \\
$\$ 367,425$ & $\$ 523,717$ \\
\hline$\$ 523,717$ & $\$ 774,371$ \\
\hline \hline
\end{tabular}


Table 6 - Historical Ratio Analysis

Profitability

Sales Growth

Gross Margin

EBITDA Margin

Net Operating Margin

ROE

ROA

\section{Asset Efficiency}

Accounts Receivable Turnover

Accounts Receivable Days on hand

Inventory Turnover

Inventory Days on hand

PP\&E Turnover

Liquidity \& Leverage

Current Ratio

Quick Ratio

Debt-to-Equity

Total Liabilities-to-equity

Cash Flow

Free Cash Flow

EBITDA Interest Coverage

\begin{tabular}{rrrr} 
& $\mathbf{2 0 0 5}$ & $\mathbf{2 0 0 6}$ & $\mathbf{2 0 0 7}$ \\
\hline NA & & $19.3 \%$ & $-14.4 \%$ \\
& $56.7 \%$ & $60.9 \%$ & $56.5 \%$ \\
$12.3 \%$ & $15.9 \%$ & $24.5 \%$ \\
$3.2 \%$ & $7.7 \%$ & $13.2 \%$ \\
$40.3 \%$ & $65.1 \%$ & $53.1 \%$ \\
$6.8 \%$ & $15.4 \%$ & $17.3 \%$
\end{tabular}

\section{3}

13.9

$31.6^{\top}$

11.6

8.2

5.0
4.8

$362.6 \%$

$448.8 \%$

N/A

10.34

$\$ 196,128$

19.14

$$
4.1
$$

$$
4.0
$$

$186.2 \%$

$260.2 \%$
20.7

17.6

26.8

13.6

11.5

11.0

4.8

4.7

$123.6 \%$

$176.4 \%$

$\$ 232,382$

16.08 